\title{
Pediatric Complex Metacarpophalangeal Joint Dislocation of the Index Finger
}

\author{
Gonzalo Sumarriva, MD, ${ }^{1,2}$ Brandon Cook, MD, ${ }^{1}$ Gustavo Godoy, MD, ${ }^{1}$ Sean Waldron, MD ${ }^{1,2}$ \\ ${ }^{1}$ Department of Orthopedics, Ochsner Clinic Foundation, New Orleans, LA ${ }^{2}$ The University of Queensland School of Medicine, Ochsner \\ Clinical School, New Orleans, LA
}

\begin{abstract}
Background: Metacarpophalangeal (MCP) joint dislocations are the result of a hyperextension injury. Complex MCP joint dislocations-those that are irreducible to closed maneuvers and require surgical intervention-are considered uncommon, even in the pediatric population. Although several structures have been identified that contribute to irreducible dislocations, the volar plate is the most significant barrier to reduction through interposition into the MCP joint. Key differences that require consideration for MCP joint dislocations in pediatric patients include ligamentous laxity, the absence of sesamoid bones, the possibility for cartilage fractures, and the possibility of growth arrest. Open surgical intervention for a complex MCP joint dislocation is performed through either the volar or dorsal approach. Controversy exists about which approach is superior. Case Report: We present the case of a 7-year-old female who sustained a complex MCP joint dislocation of the index finger. After numerous unsuccessful attempts at closed reduction, the patient underwent open reduction through the dorsal approach. The phalangeal head had buttonholed through the volar plate and was reduced by using a Freer elevator as a lever and applying gentle traction and flexion. At 4-week follow-up, the patient was pain-free and had regained nearly full range of motion of the index finger MCP joint.

Conclusion: In addition to the classic volar and dorsal approaches, different techniques have been used to reduce complex dislocations in pediatric patients, including arthroscopic surgery, a percutaneous technique with manipulation of a skin hook, and a percutaneous technique with a dorsal incision. As demonstrated in this case, open reduction through the dorsal approach remains a viable treatment option for complex MCP joint dislocations in the pediatric population.
\end{abstract}

Keywords: Joint dislocations, metacarpophalangeal joint, open reduction, palmar plate

Address correspondence to Sean Waldron, MD, Department of Orthopedics, Ochsner Clinic Foundation, 1514 Jefferson Hwy., New Orleans, LA 70121. Tel: (504) 842-3970. Email: swaldron@ochsner.org

\section{INTRODUCTION}

The mechanism of injury of a metacarpophalangeal (MCP) joint dislocation is typically a hyperextension after a fall onto an outstretched hand. MCP joint dislocations can occur with the proximal phalanx dislocating dorsally or volarly, with the dorsal dislocation occurring significantly more often. ${ }^{1}$ MCP joint dislocations that can be reduced using closed techniques are known as simple dislocations, while those that are irreducible to closed maneuvers and require surgical intervention are known as complex dislocations. Simple dislocations can be reduced via closed technique in which the wrist and proximal interphalangeal joint are flexed while a translational force is applied to the base of the proximal phalanx. ${ }^{1}$ Complex dislocations are considered rare occurrences. ${ }^{2}$ The anatomy of complex dislocations involves multiple structures, but several studies have shown the volar plate to be the most important structure interfering with reduction, typically through interposition into the MCP joint. $^{2}$
Two well-recognized surgical reduction approaches exist: the dorsal approach and the volar approach. Controversy exists about which surgical approach is superior. ${ }^{1,2}$ Proponents of the dorsal approach believe that the dorsal approach carries less risk to the digital neurovascular bundle and allows repair of osteochondral defects. Advocates of the volar approach believe the volar approach allows for improved viewing of the pathologic anatomy and also for repair of the volar plate, which may lead to improved longterm stability.

We report the case of a 7-year-old patient who sustained a complex index finger MCP joint dislocation.

\section{CASE REPORT}

A 7-year-old female with no significant medical or surgical history sustained a complex index finger MCP joint dislocation after being kicked in her right hand by another child. The patient initially presented to an outside hospital, and 3 different physicians unsuccessfully attempted reduction 

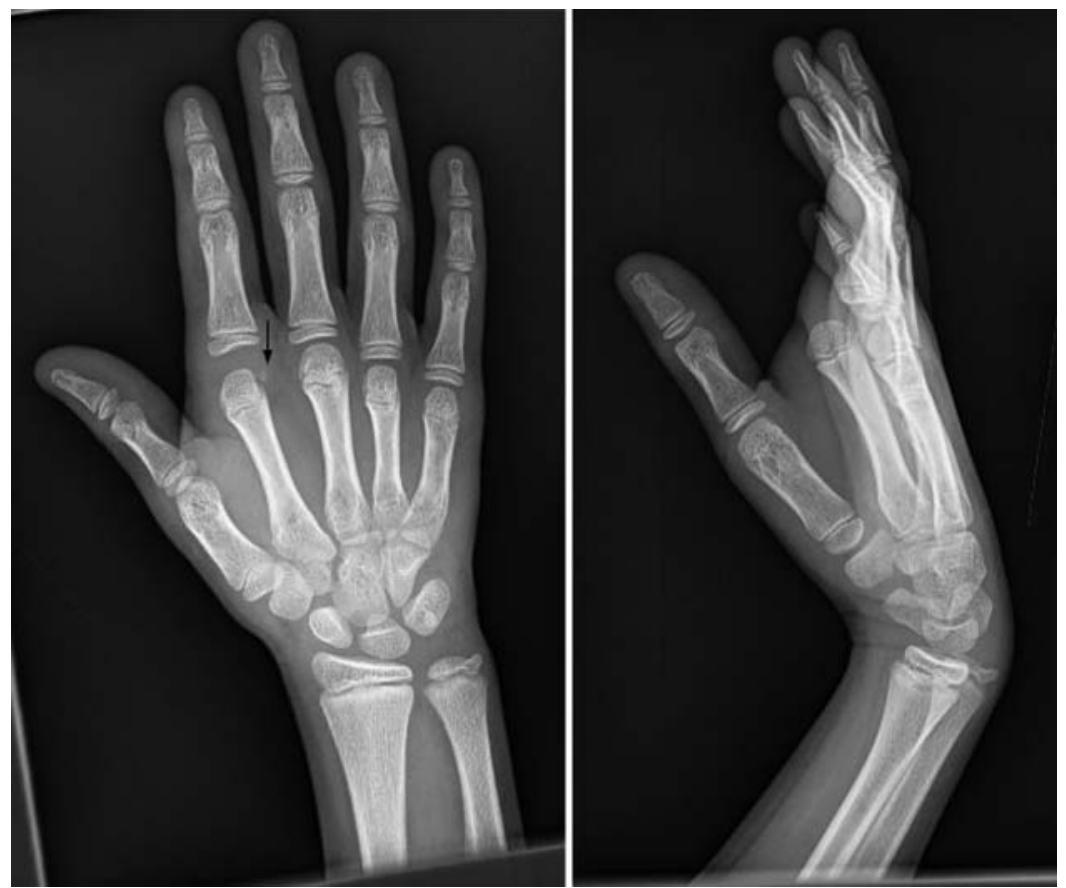

Figure 1. Right hand anteroposterior (left) and lateral (right) radiographs preoperatively demonstrate dorsal dislocation of the index metacarpophalangeal joint with a widened joint space and a small avulsion fracture of ulnar collateral ligament (arrow).

under digital block anesthesia. She was transferred to our facility for further management.

On physical examination, the patient had swelling, deformity, and tenderness to palpation at the second MCP joint. The patient denied paresthesia. Sensation and capillary refill time were normal over the finger. Plain-film radiographs verified dislocation of the right index MCP joint with a widened joint space and a small avulsion fracture of the ulnar collateral ligament (Figure 1). The patient was sedated, and an orthopedic surgeon made another unsuccessful attempt to reduce the dislocation in the emergency department (ED). After being informed of the risks, benefits, and alternatives of surgical treatment, the patient's parents agreed to surgery.

Closed reduction under fluoroscopy was attempted unsuccessfully in the operating room, so the MCP joint of the right index finger was opened. A longitudinal incision was made over the dorsal aspect of the MCP joint with a gentle ulnar curve over the joint itself. The extensor mechanism was split in line with its fiber, and the joint capsule was identified and split longitudinally. Once adequately exposed, the phalangeal head was found to have buttonholed through the volar plate. Using a Freer elevator (Sklar Surgical Instruments) as a lever and applying gentle traction and flexion, the proximal phalanx head was reduced through the volar plate. A small osteochondral fragment was found and affixed to the distal ulnar metacarpal head using 4-0 Vicryl suture (Ethicon, Inc.). The extensor mechanism, subcutaneous layer, and skin were closed using appropriate sutures. Reduction was confirmed with fluoroscopy. The patient was placed in a volar splint with approximately $45^{\circ}$ of flexion and discharged on postoperative day zero without any complications.

The patient was seen in clinic at 2-week follow-up with stiffness through the range of motion and was switched from splint to brace and buddy tape. At 4-week follow-up, the patient had painless, nearly full range of motion of the index MCP joint. Radiographs confirmed a well-reduced MCP joint with no evidence of the previous ulnar collateral ligament avulsion fracture (Figure 2).

\section{DISCUSSION}

The MCP joint is a synovial joint that involves motion in multiple planes. The complex shape of the metacarpal head, in addition to the articular surface and supporting soft tissue structures, allows for flexion, extension, adduction, and circumduction. ${ }^{1}$ The MCP joint is enclosed in a joint capsule that extends from the metacarpal neck to the base of the proximal phalanx. The joint is reinforced by ligamentous structures on all sides. The volar plate is present on the palmar aspect of the MCP joint and is a rectangular reinforcement of the capsule composed of a thick fibrocartilaginous portion distally and a looser membranous portion proximally. ${ }^{1,3}$

MCP joint dislocations are relatively uncommon ${ }^{4}$ and occur less often than interphalangeal joint dislocations. ${ }^{1}$ MCP joint dislocations most frequently affect the index finger, followed by the thumb and little finger. The border digits of the index and little finger are prone to dislocation because of increased vulnerability to trauma and lack of stabilization by 2 adjacent deep transverse metacarpal ligaments. ${ }^{1,5}$ The suggested reduction technique for a simple MCP joint dislocation is flexion at the wrist and proximal interphalangeal joint to relax flexor tendons while pressure is applied to the proximal phalanx at the base. ${ }^{1}$ Simple distraction or extension maneuvers for reduction of MCP joint dislocations are typically unsuccessful and can convert a reducible dislocation to an irreducible one because traction on the joint may draw dorsally and 


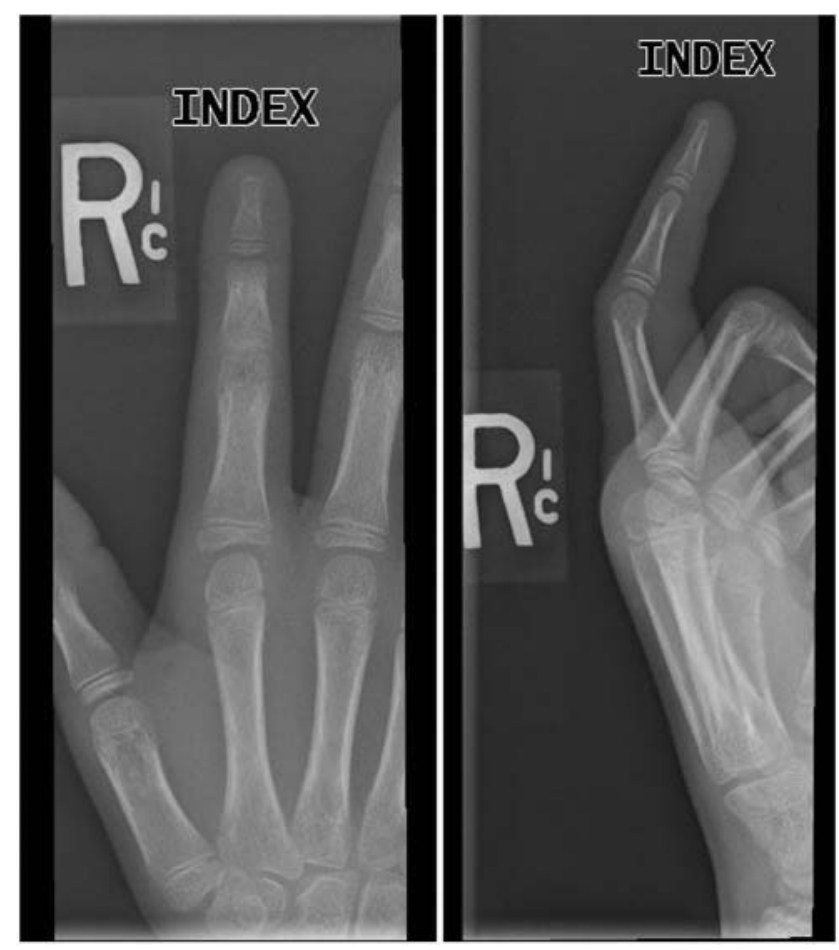

Figure 2. Right index finger anteroposterior (left) and lateral (right) radiographs at 4-week follow-up demonstrate a well-reduced index metacarpophalangeal joint.

completely fold the volar plate into the joint. ${ }^{1,3}$ In addition, the ligamentous laxity found in children may allow conversion from simple to complex dislocation. ${ }^{3}$

In complex dorsal MCP joint dislocations, the volar plate has been identified as the most significant barrier to reduction, ${ }^{2}$ typically through interposition into the MCP joint. Finger hyperextension may lead to rupture of the volar plate at its weakest point, the proximal membranous attachment to the metacarpal metaphysis and periosteum. The metacarpal head becomes displaced toward the palm, while the volar plate, attached to the phalanx, slips into the MCP joint and becomes trapped. ${ }^{3}$ The principal problem becomes the volar plate that is caught between the base of the proximal phalanx and the metacarpal head. ${ }^{3}$ As with our patient, the metacarpal head may also buttonhole through the palmar structures and become entrapped distally by the natatory ligament, proximally by the superficial transverse metacarpal ligament, and laterally by the flexor tendon and lumbrical muscle. ${ }^{1,6}$ These structures together form a tendinous noose around the metacarpal neck that becomes tight during closed reduction maneuvers. ${ }^{1,4}$

Volar MCP joint dislocations can occur with either hyperflexion or hyperextension injuries ${ }^{1}$ and are less common than dorsal MCP join dislocations. In addition, different structures are involved in complex volar MCP joint dislocations, including the dorsal capsule, distal insertion of the volar plate, and the tendinous junction connecting the fourth and fifth extensor digitorum communis tendons. ${ }^{1}$

Radiographs can be used to confirm the diagnosis of complex MCP joint dislocations and may show a widened joint space or a sesamoid bone trapped in the joint, a pathognomonic sign for complex dislocation. ${ }^{1}$ Concomitant fractures may occur at the base of proximal phalanx or the metacarpal head and occur in half of MCP joint dislocations. ${ }^{1}$

Complex pediatric MCP joint dislocations occur in a similar fashion as those in adults, with differences existing in the absence of sesamoid bone formation and the potential for cartilage fractures on the metacarpal head at the collateral ligament insertions. ${ }^{5}$ Before mid-adolescence, the sesamoid bones may not be ossified; therefore, sesamoid interposition into the MCP joint may not be an issue or at least will not be evident on radiographs. ${ }^{5}$ Children have a higher tendency for variable physeal and epiphyseal fractures rather than ligamentous injuries that may occur in adults. ${ }^{3}$ As in the case of our patient, the soft tissue sleeve may pull off small osteochondral fragments from the ulnar side of the metacarpal, as the ulnar side is more susceptible to biomechanical stresses. ${ }^{3}$

Surgical treatment for complex MCP joint dislocations includes 2 commonly used approaches. Farabeuf ${ }^{7}$ first described the dorsal approach in 1876, while Kaplan ${ }^{6}$ described the volar approach in 1957. Each approach has advantages and disadvantages, and controversy exists about which approach is superior. Barry et al recommended the volar approach for experienced hand surgeons as it allows for a complete anatomic restoration of the joint to be achieved, but they recommended the dorsal approach for the infrequent hand surgeon as it is a simple, safe choice with apparently stable results. ${ }^{2}$

The volar approach allows direct access to the lesion and visualization of the ligaments and tendons that can trap the metacarpal head. ${ }^{4,8}$ The volar approach also allows repair of the volar plate, ${ }^{4}$ which may decrease the risk of late instability. An MCP joint dislocation displaces the neurovascular bundle superficially and immediately under the skin, ${ }^{1}$ placing it at risk in the volar approach. In particular, the palmarly displaced radial neurovascular structures are susceptible to injury in the index and long finger, while the ulnar digital nerve and artery are at risk in the ring and little finger. ${ }^{4}$

In contrast, the dorsal approach allows repair of osteochondral fractures and avoids the digital neurovascular bundle, the primary reasons this approach was chosen for our patient. The dorsal approach allows excellent exposure for evaluation of the volar plate that may become tented over the metacarpal head. ${ }^{4}$ To aid in reduction, a volar plate incision is possible but unrepairable. ${ }^{3,9}$ Although an unrepaired volar plate poses a theoretical risk of late instability, this complication has not been reported for the dorsal approach. ${ }^{2}$

After surgical reduction, the joint should be immobilized in a functional position for less than 3 weeks. ${ }^{4}$ Longer immobilization may lead to degenerative arthritis or reduced range of motion. ${ }^{4}$ Light and Ogden's slightly different recommendation for early protected mobilization is using a dorsal splint to prevent full extension. ${ }^{3}$

Complications of general MCP joint injury include degenerative arthritis from repeated closed reduction attempts, traumatic open reduction or prolonged dislocation, osteonecrosis of the metacarpal head, and stiffness. ${ }^{1}$ In children, a unique complication is growth arrest that may occur as a consequence of vascular damage and lead to limited joint motion, a shortened metacarpal, or deformity of the metacarpal head. ${ }^{3}$ The metacarpal head is intracapsular with small vessels that supply the epiphysis and physis. These vessels are susceptible to injury in fractures of the distal metacarpal. $^{3}$ 
New, less invasive techniques have been described in addition to the 2 common approaches: arthroscopic reduction and 2 percutaneous techniques. Kodama et al described a case of arthroscopic reduction of the index finger in an 11year-old male. ${ }^{8}$ Reduction was achieved by using a probe to push the torn proximal attachment of the volar plate palmarly while the metacarpal head was pushed dorsally. Because arthroscopy uses only two $5 \mathrm{~mm}$ portals, it may damage structures less than open surgery but only allows vision of the intraarticular anatomy, which may limit repair of extraarticular pathology, including the volar plate or osteochondral fractures. ${ }^{8}$ Boden et al described a percutaneous technique using a skin hook to reduce a complex MCP joint dislocation of the index finger of a 8-year-old female. ${ }^{10}$ Kaplan described dimpling of the skin over the proximal palmar crease as a pathognomonic sign of dorsal complex MCP joint dislocation. ${ }^{6}$ This puckering sign was used for placement of a skin hook. Traction was applied to the volar structures via the skin hook and resulted in successful reduction on first attempt. The authors stated that the traction may have moved the obstructing palmar structures forward and allowed for closed reduction. The closed and simple manner of reduction obtained using this technique suggests its use prior to more invasive procedures. Sodha et $\mathrm{al}^{11}$ described another percutaneous technique using a dorsal incision for reduction of complex MCP joint dislocations in 4 patients ages $6,8,16$, and 24 years. An incision was made just lateral to the extensor tendon onto the dorsum of the metacarpal neck proximal to the base of the proximal phalanx. The incision divided the volar plate and allowed for successful reduction on first attempt in all 4 cases. The procedures were performed under local anesthesia and in the operating room ( 3 cases) or the ED ( 1 case). The technique has the advantage of limited risk to neurovascular structures. It also avoids the risks associated with open surgery. Similar to the dorsal approach, none of the 3 novel techniques allows repair of the volar plate, which may lead to a theoretical risk of late joint instability.

The arthroscopic and the percutaneous skin hook technique were only reported in one patient each, raising the question of whether similar results would be obtained from repeat situations. Another disadvantage of the 2 percutaneous techniques is that the procedures were performed without visualization of the pathologic joint anatomy. ${ }^{8}$ On the other hand, the percutaneous techniques are quick, can be performed in the ED under local anesthesia, and may save the patient from an open operation and anesthesia. Although information is limited, the percutaneous techniques may be worth considering in complex MCP joint dislocations.

\section{CONCLUSION}

Complex dorsal MCP joint dislocations occur after hyperextension injuries and are rare occurrences, in both adults and children. Pediatric MCP joint dislocations are similar to adult dislocations, but unique differences in children are ligamentous laxity that allows conversion from simple to com- plex dislocation during reduction maneuvers, absence of sesamoid bones to interpose into the joint, possible cartilage fractures, and possible growth arrest after vascular damage. The dorsal approach and the volar approach are the most common surgical techniques used to reduce these dislocations, although controversy exists regarding which is preferable. In addition to open reduction, less invasive techniques performed on pediatric patients have been described, including arthroscopic surgery and 2 percutaneous techniques, the first using a skin hook and the second using a dorsal incision to release the volar plate. As our case shows, open reduction through the dorsal approach is a viable treatment option for complex MCP joint dislocations in children.

\section{ACKNOWLEDGMENTS}

The authors have no financial or proprietary interest in the subject matter of this article.

\section{REFERENCES}

1. Dinh $P$, Franklin A, Hutchison B, Schnall SB, Fassola I. Metacarpophalangeal joint dislocation. J Am Acad Orthop Surg. 2009 May;17(5):318-324.

2. Barry K, McGee H, Curtin J. Complex dislocation of the metacarpo-phalangeal joint of the index finger: a comparison of the surgical approaches. J Hand Surg Br. 1988 Nov;13(4):466468.

3. Light TR, Ogden JA. Complex dislocation of the index metacarpophalangeal joint in children. J Pediatr Orthop. 1988 May-Jun;8(3):300-305.

4. Durakbasa O, Guneri B. The volar surgical approach in complex dorsal metacarpophalangeal dislocations. Injury. 2009 Jun;40 (6):657-659. doi: 10.1016/j.injury.2008.10.027.

5. Türker T, Sheppard JE. Emergency open reduction for an irreducible dislocation of the metacarpophalangeal joint of the thumb in a child. J Hand Microsurg. 2015 Jun;7(1):166-169. doi: 10.1007/s12593-014-0135-5.

6. Kaplan EB. Dorsal dislocation of the metacarpophalangeal joint of the index finger. J Bone Joint Surg Am. 1957 Oct;39-A (5):1081-1086.

7. Farabeuf LHF. De la luxation du ponce en arrière. Bull Soc Chir. $1876 ; 11: 21-62$.

8. Kodama A, Itotani Y, Mizuseki T. Arthroscopic reduction of complex dorsal metacarpophalangeal dislocation of index finger. Arthrosc Tech. 2014 Apr 3;3(2):e261-e264. doi: 10.1016/j. eats.2013.11.008.

9. Becton JL, Christian JD Jr, Goodwin HN, Jackson JG 3rd. A simplified technique for treating the complex dislocation of the index metacarpophalangeal joint. J Bone Joint Surg Am. 1975 Jul;57(5):698-700.

10. Boden RA, Cavale N, Fleming AN. Kaplan's puckering sign in complex dorsal dislocation of the metacarpophalangeal joint of the index finger. J Hand Surg Br. 2006 Oct;31(5):576-577.

11. Sodha S, Breslow GD, Chang B. Percutaneous technique for reduction of complex metacarpophalangeal dislocations. Ann Plast Surg. 2004 Jun;52(6):562-565; discussion 566.

This article meets the Accreditation Council for Graduate Medical Education and the American Board of Medical Specialties Maintenance of Certification competencies for Patient Care and Medical Knowledge. 